\title{
Preventive aspects of genetic morbidity: experiences of the Canadian model
}

\author{
M.M. Khalifa'
}

SUMMARY Genetic disorders are emerging as a major health problem in industrialized countries, especially with greater control of environmental diseases. Of total admissions to major paediatric hospitals, $50 \%$ are due to genetic disorders, and at least $10 \%$ of all adult admissions to hospitals involve major genetic contributions. Several programmes aimed at preventing or ameliorating these genetic disorders are being implemented. Identifying people at risk of genetic disease has helped decrease the burden of such diseases on families and society. Early recognition also leads to greater success of treatment and improves outcome and prognosis. In Arab populations, genetic disorders are still not perceived as a major health problem, even though they are widely prevalent. Applying similar programmes of early detection, maternal screening, neonatal screening, carrier lesting and susceptibility testing will significantly help reduoe tho impaot of these dieordere in our populations.

\section{Introduction}

There is strong evidence that genetic factors play an important role in human diseases [I]. In the technologically develoned countries, genetic disorders and birth defects are emerging as a major health problem, especially after eliminating malnutrition and endemic diseases, and having better control over epidemic and environmental disorders. There are more than 6000 single-gene Mendelian disorders described. Of total deliveries, $16 \%$ involve birth defects, and almost $50 \%$ of these are major (i.e. they have medical, surgical or cosmetic consequences) [2]. About $50 \%$ of all paediatric admissions to major hospitals involve birth defects or genetic diseases. After accidents, they are the leading cause of death in children in North America [2]. In the United States of America, 250000 births yearly involve major birth defects, and over 10 million American men and women currently have birth defects. In Canada, it is estimated that $60 \%$ of Canadians will experience a disease with a significant genetic component in their lifetime [3].

Until now, the prevailing concept of medicine is that of the body as a machine, which the doctor is called upon to fix when it breaks [4], similar to the role of an engineer who uses technology in fixing a disabled machine $[5,6]$. This engineering mentality has dominated medical practice for centuries, and has influenced medical teaching and shaped the provision of medical care. This model is dependent on ad-

'Division of Medical Genetics, Department of Paediatrics, Queen's University, Kingston, Ontario, Canada. Receivad: 10/09/98; accepted: 02/09/9g

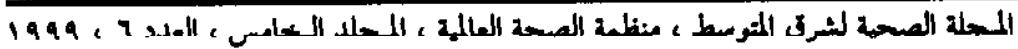


vanced and expensive technology, but the escalating cost of providing such treatment has made it prohibitively expensive. It is estimated that the lifelong cost of treating a child with moderate mental retardation (MR) runs to millions of US dollars and it is considerably more for those with severe MR. Medicine is now gradually moving into a new era and new territory - an era of preventive medicine, which entails identifying people at risk of developing diseases and trying to prevent the occurrence of these diseases, or ameliorate or alter their course before they occur. Genetics is leading medicine in this area.

In some technologically developed countries, several programmes are currently in place to identify individuals at risk of genetic disorders amenable to prevention or treatment. As technology evolves, newer programmes and services are being introduced, once their merit becomes evident. Some of these programmes are mandated by law, others are voluntary.

In Canada, where health services are constitutionally guaranteed to each individual, the preventive genetic programmes are universally available to everyone. The most significant measure is genetic screening and Canada has been a leader in taking the initiative to apply the principles of genetic screening in the health care system [7]. The currently available services in Canada to identify at-risk individuals for genetic disorders through genetic screening include:

- newborn screening

- maternal serum screening

- carrier screening

- presymptomatic testing

- susceptibility testing

- prenatal testing

- preimplantation testing.

\section{Genetlc screening}

Genetic screening is the monitoring of a population to identify affected fetuses, or to determine those members of the population who, despite being apparently normal, have genotypes that are associated with diseases, or may lead to diseases in their offspring $[7,8]$. Genetic screening thus serves several objectives. First, it can lead to therapy, as in the case of newborn screening which aims for the earliest possible recognition of disorders so that intervention can prevent the serious consequences of these diseases. Secondly, screening can identify those whose pregnancies are at increased risk of producing offspring with serious genetic abnormalities. They can then be counselled on their reproductive options, including prenatal diagnosis and treatment.

\section{Newborn screening}

Newborn screening is the most widely used form of genetic testing in Canada and the United States. Currently, screening is conducted for the following 15 metabolic and non-metabolic diseases.

- biotinidase deficiency

- congenital adrenal hyperplasia

- congenital hypothyroidism

- cystic fibrosis

- galactosaemia

- haemoglobinopathies

- homocysteinuria

- maple syrup urine disease

- phenylketonuria

- tyrosinaemia

- congenital toxoplasmosis

- congenital hearing defect

- human immunodeficiency virus 
Table 1 Newborn screening in Canada, by province and type of blood screening, 1990

\begin{tabular}{|c|c|c|c|c|c|c|c|c|}
\hline Province & PKU & Thyr & AAC & Gal & MDys & CAH & Biot & Tyr \\
\hline British Columbia & $a+$ & + & & + & & & & + \\
\hline Alberta & & + & + & + & & & & \\
\hline Saskatchewan & + & + & & & & & & \\
\hline Manitoba & + & + & + & + & + & & + & + \\
\hline Ontario & & + & + & & & & & \\
\hline Quebec & & + & + & & & & + & + \\
\hline Nova Scotia & + & + & & & & & & \\
\hline Now Brunswick & + & + & & & & & & \\
\hline Prince Edward & + & + & & & & & & \\
\hline Newtoundland & + & + & + & & & & & + \\
\hline
\end{tabular}

Urine screening is used at 2-4 weeks for AAC in Quebec (95\%) and Manitoba (85\%).

Pilot studies are being conducted in Quebec to screen for neuroblastoma and in British Columbia to screen for cystic fibrosis.

FKU = pnenyikeronuria

Gal = galactosaemia

Biot $=$ biotinidase deficiency

Thyr $=$ tiypothyroidism

MDys = muscular dystrophy

Tyr $=$ tyrosinaemia
AAC - amino aoid ohromatogram

$C A H=$ congenital adrenal hyperplasia
- muscular dystrophy

- neuroblastoma

Depending on the province (Canada) or state (the United States), virtually all neonates are screened for between 2 and 10 genetic and non-genetic conditions. The newborn screening programme in Canada. by province, is summarized in Table 1 . The combined number of infants screened in the neonatal period, and the number of neonatal screening tests performed, far exceed those for any other type of genetic test [9].

In Ontario, the largest Canadian province (population approximately 11 million), all neonates are screened for two metaholic diseases, phenylketonuria (PKU) and hypothyroidism. Over 4 million infants have been screened for PKU since the implementation of the programme in July. 1965. Only one false negative result has been confirmed in this time [10]. The incidence of PKU and its variants in Ontario is
$1 / 11700$ and is $1 / 21300$ for classic PKU. In keeping with the Canadian Health Act mandate to provide affordable treatment to all citizens, the Ministry of Health policy is to pay for the special treatment and food products required by patients identified through the screening programme. Current$1 y, 459$ patients in Ontario are under treatment, 225 with PKU and 234 for other metabolic diseases (unpublished data). The annual cost of such a programme in Ontario is approximately 1 million Canadian dollars, making it a very cost-effective programme.

\section{Maternal serum screening}

Down syndrome (DS) is the most eommon chromosome abnormality in humans. It represents about one-third of all cases of mental retardation. Trisuny 18 and 13 are rare, and usually not compatible with life. Because the risk of having a child with tri-

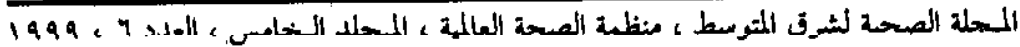




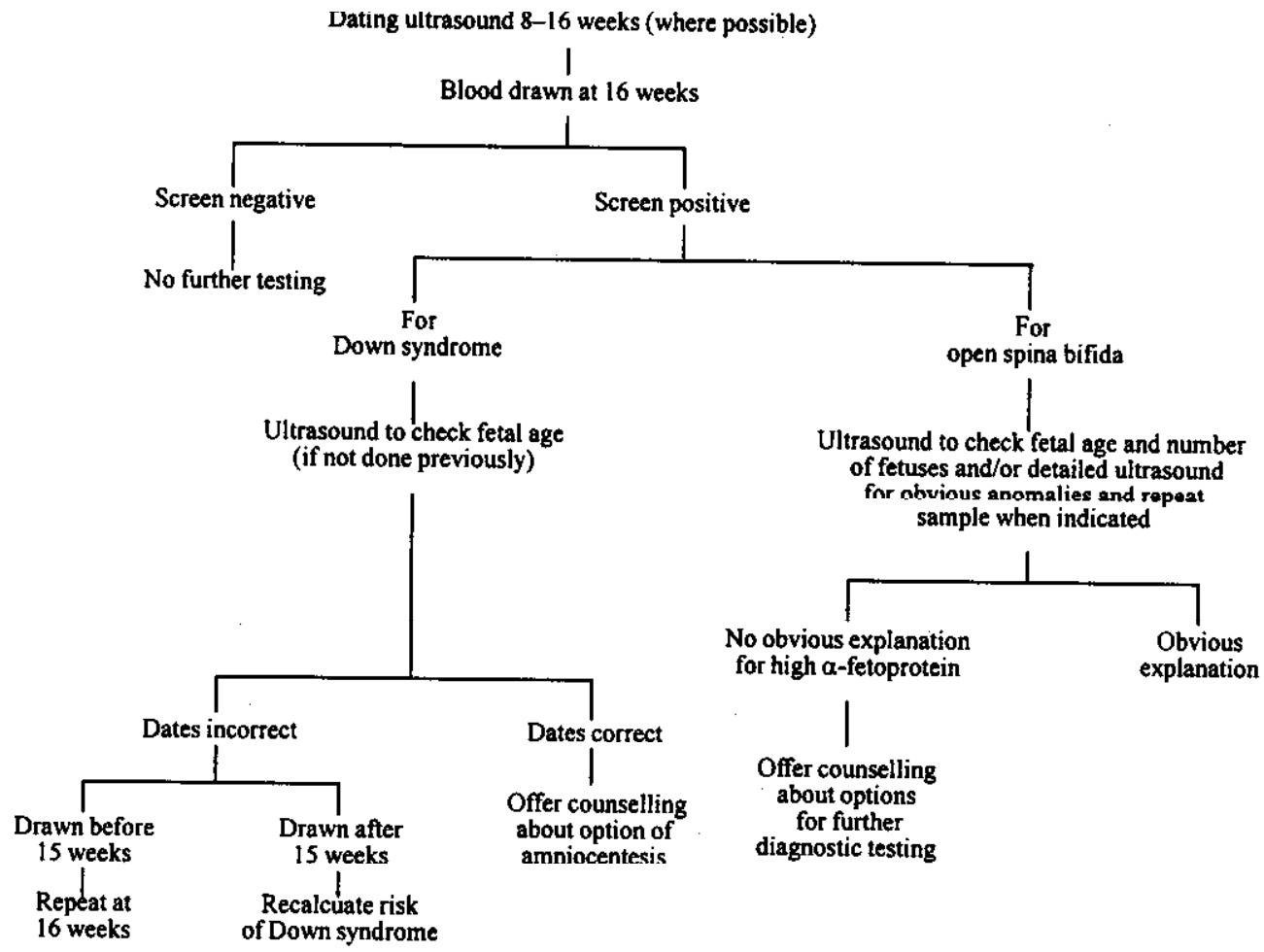

Figure 1 Maternal serum screening protocol in Ontario, Canada

somy increases with advanced maternal age, pregnancies of mothers $\geq 35$ years of age are screened by amniocentesis or chorionic villus sampling. If all mothers 35 years or older took part in prenatal diagnosis, theoretically only $33 \%$ of all DS could be detected, as two-thirds of all DS occurs in younger mothers. By screening all pregnant women by measuring $\alpha$-fetoprotein (AFP), unconjugated estriol, (uE3) and human chorionic gonadotropin (hCG), about $70 \%$ of all DS (Table 2) and $80 \%$ of neural tube defects (NTD) and of trisomy 18 could be detected $[I I]$. This makes maternal serum screening the most significant screening test for preventing birth defects.

Commencing on 1 July, 1993, the Ministry of Health in Ontario funded a prov- ince-wide programme for screening all pregnant women for AFP, uE3 and hCG between the 15th and 20th weeks of pregnancy. These measurements, used in conjunction with maternal age, provided a risk estimate of having a pregnancy with DS,

Table 2 Screening for Down syndrome

\begin{tabular}{lcc}
\hline Modality & $\begin{array}{c}\text { Detection } \\
\text { rate }(\%)\end{array}$ & $\begin{array}{c}\text { False-positive } \\
\text { rate (\%) }\end{array}$ \\
\hline Age $\geq 35$ years & 33 & 10.0 \\
Age + AFP & 40 & 12.5 \\
Age + 2 markers & 08 & 8.6 \\
Age + 3 markers & 72 & 8.0 \\
\hline
\end{tabular}


Table 3 Cost-effectiveness of maternal serum screening (MSS) in Ontario

\begin{tabular}{lccc}
\hline Screening & $\begin{array}{c}\text { Cost } \\
\text { (millions Canadlan dollars) }\end{array}$ & No. of amniocenteses & $\begin{array}{c}\text { Detection rate } \\
\text { (\%) }\end{array}$ \\
\hline Age alone $\geq 35$ years & 15.00 & 15000 & 33.0 \\
MSS for all & 12.00 & 8437 & 72.0 \\
MSS for $\geq 35$ years & 0.52 & 3000 & 26.0 \\
$\begin{array}{l}\text { MSS for < 35 years + } \\
\text { amniocentesis for } \geq 35 \text { years }\end{array}$ & 19.70 & 19725 & 79.5 \\
\hline
\end{tabular}

Table 4 Down syndrome/amnlocentesis ratio

\begin{tabular}{lc}
\hline Screening & $\begin{array}{c}\text { Down } \\
\text { syndrome } \\
\text { cases/ } \\
\text { amnlocenteses }\end{array}$ \\
\hline Age alone > 35 years & $1 / 150$ \\
Ago + AFP & $1 / 80$ \\
Age + 3 markers & $1 / 37$ \\
\hline
\end{tabular}

$A F P=\alpha$-fetoprotein

NTD or trisomy 18 for every woman screened. Figure 1 illustrates the protocol used for maternal serum screening in Ontario. This programme has not only proved to be cost-effective (Table 3), but has also decreased the number of amniocenteses performed for every DS detected, and with a higher detection rate (Table 4). Currently the efficacy of first trimester screening by measuring other biochemical markers such as PAPPA-A and -B subunit of $h \mathrm{CG}$ as well as the ultrasound measurement of nuchal translucency is being studied.

\section{Carrler screening}

There are over 400 inborn errors of metabolism in humans with identifiable biochemical or genetic abnormalities [12], the vast majority of which are recessively inherited.
Carrier detection of most of these disorders is achievable either by biochemical or immunological methods, or more recently by direct DNA mutation analysis or indirect linkage study using polymorphic DNA markers flanking the disease locus in informative families. Identifying carriers among high-risk families not only allows them informed reproductive choice, it may also have therapeutic or preventive health benefits. People with sickle-cell trait should be aware of the possible hazards of reduced ambient oxygen concentrations, such as in high altitudes or mountain climbing. Also, altering anaesthesia could avert inadvertent hypoxia that might be particularly hazardous to such people. Similarly, heterozygosity for $\alpha_{1}$-antitrypsin deficiency may predispose an individual to chronic pulmonary disease. Avoidance of tobacco smoke and other noxious inhalants may reduce this risk. Population screening for carriers is, however, better limited to groups defined as high-risk for specific diseases, such as screening for Tay-Sachs disease among Ashkenazi Jews.

\section{Presymptomatic testing}

Certain autosomal dominant diseases with variable expressivity or late-age of onset, such as Huntington disease, spinocerebellar laratrophy, familial amyotrophic lateral 
sclerosis and Alzheimer disease, have a propensity for neurodegenerative manifestations. DNA testing now makes it possible to identify people who are subtly affected or asymptomatic, but who will ultimately develop the disease. Testing these asymptomatic people might be problematic. There is no real medical benefit, as there is no effective intervention to prevent, delay or alter the onset of these disorders. However, it allows for more informed reproductive, and other life, decisions. For these reasons, pretest and post-test counselling should be provided with ongoing support, whatever the decision regarding testing or not, or its outcome.

\section{Susceptibility testing}

Presymptomatic testing for Huntington disease or other neurodegenerative disorders can alter the status of "at-risk" individuals to either "not at-risk" or "affected". We now know that a growing number of other diseases (e.g. breast and colon cancer, LiFraumeni syndrome or thrombophilia, coronary heart disease) have major genetic components. Testing for these diseases can identify individuals with high susceptibility for developing these conditions. Susceptibility testing could allow for intervention for the at-risk individuals, such as prophylactic surgical intervention (in cancer), or therapeutic intervention (as in folic acid supplementation for familial thrombophilia).

\section{Preimplantation diagnosis}

Currently, prenatal diagnosis for high-risk families is carried out in the post-implantation period. During the past 10 years, the development of three technologies, in vitro fertilization, micromanipulation of gametes and embryos, and ultra microbiochemical techniques for gene mutation analysis, now enables the diagnosis of genetic diseases in the preimplantation period $[13,14]$.
The possibility of in vitro diagnosis of genetic diseases is intriguing. It would eliminate the conflict over termination of pregnancy for some individuals on moral, religious and personal grounds, because genetic testing could be carried out prior to implantation. However, much work is needed to ensure that genetic analysis is unequivocally accurate, and that the risk of all procedures involved is low.

\section{Can preventive genetic measures be applied to Arab populations?}

A majority of the Arabic countries have the expertise and resources to apply most of these preventive measures, especially in the areas of newborn screening and carrier screening for prevalent genetic disorders. However, having the technology and resources alone are not enough to start effective programmes. There are certain principles and prerequisites needed for any successful genetic screening programme [8].

\section{Principles of genetic screening}

Screening should be voluntary. Unless mandated by law, screening for genetic diseases should be voluntary and with the patient's informed consent. The patient might not wish to face the dilemma of choosing from the several options that may result from the screening process $[7,8]$. Newborn screcning for metabolic diseases is, however, legally required in most provinces and states.

Sereening should not be expected to detect all patients. It is unrealistic to expect any screening programme to identify all affected individuals. Maternal serum screening, for example, identifies approximately $70 \%$ of DS, $80 \%$ of NTD and $80 \%$ of trisomy 18. Avallability of technology does 
not of itself justify screening. Identifying all chromosomal abnormalities and almost all inborn errors of amino acid metabolism can be done in the neonatal period. However, screening is recommended only for those disorders that fulfill the essential prerequisites for screening programmes $[7,8]$.

\section{Prerequisites for genetic screening} Ability to alter the outcome

Population screening is usually performed only if the abnormal finding can change the clinical management, and consequently result in a favorable outcome. Neonates are screened for those metabolic diseases that can be treated, such as PKU and hypothyroidism, but not for those disorders that are untreatable, such as mucopolysaccharidosis or Tay-Sachs disease. Early detection of sickle-cell anaemia has recently been shown to reduce the morbidity and mortality of this disorder [14]. Newborn screening for this disorder among Arab populations where the disease is highly prevalent should warrant serious evaluation and consideration.

\section{Cost-effectiveness}

Not every treatable condition is, or should be, screened. For unly a few disorders does the cost of screening justify the financial and emotional savings of detecting the affected individuals. Although newborn screening is generally applied for several diseases, only screening for PKU, hypothyroidism, and probably sickle-cell anaemia, universally satisfies the criteria for cost-effectiveness in neonatal screening.

\section{Reliable methods of assessment}

The assay to be used for screening should have a high predictive value. Because genetic disorders are individually rare, even low false-negative rates could result in a given abnormal value being more likely to be a falsepositive than a true-positive value.

\section{Ability to handle the problem}

It should be anticipated that unexpected problems will arise in any screening programme. Mechanisms should be in place to handle and deal with these inevitable problems. In most of the neonatal screening programmes in North America, advisory committees have been established to monitor the programmes and deal with such problems.

In addition, no genetic screening programme will be successful if not accompanied by extensive educational activities aimed at both the general public and health care providers. Without proper education, information and even counselling, the gencral public and health care providers may not effectively participate in these programmes.

It is very important for health professionals working among Arab populations, who have been able to identify the impact of genetic diseases on them, to start to implement some of these preventative measures. The resources and expertise are available and the population deserves such services.

\section{References}

1. Scriver CR. Human genes: determinants of sick populations and sick patients. $\mathrm{Ca}$ nadian journal of public health. Revue Canadienne de sante publique, 1988, 79:222-4.
2. Buyrse ML ed. Birth defect encyclopedia. Dover, Center for Birth Defects Information Services Incorporated, Cambridge, Massachusetts, Blackwell Scientific Publications, 1990. 
3. Baird PA et al. Genetic disorders in children and young adults: a population study. American journal of human genetics, 1988, 42:677-93.

4. Childs B. A logic of disease. In: Scriver CR et al., eds. The metabolic basis of inherited disease, 7th ed. New York, McGraw-Hill, 1995.

5. Powles J. On the limitations of modern medicine. Science, medicine, and man, 1973, 1:1-30.

6. Mckeown T. The role of medicine. London, Nuffield Trust, 1976.

7. Committee for the study of inborn errors of metabolism, genetic screening programs: principles and research. Washington DC, National Academy of Sciences, 1975.

8. Principles of screening: Report of the Committee on screening of the American College of Medical Genetics clinical practice committee. Bethesda, Maryland, American College Medical Genetics, 1997.

9. Hiller EH et al. Public participation in medical policy-making and the status of consumer autonomy: the example of newborn-screening programs in the United States. American journal of public health, 1997, 87:1280-8.

10. Hanley WB et al. Newborn phenylketonuria (PKU) Guthrie (B|A) screening and early hospital discharge. Early human development, 1997, 47:87-96.

11. Haddow JE et al. Prenatal screening for Down's syndrome with use of maternal serum markers. New England journal of medicine, 1992, 327:588-93.

12. Scriver CR et al, eds. The metabolic basis of inherited disease, 7th ed. New York, McGraw-Hill, 1995.

13. Grifo JA et al. Pregnancy after embryo biopsy and coamplitication of DNA from $X$ and $Y$ chromosomes. Journal of the American Medical Association, 1992, 268:727-9.

14. Handyside $\mathrm{AH}$ et al. Biopsy of human preimplantation embryos and sexing by DNA amplification. Lancet, 1989, 1:3479.

15. Gaston $\mathrm{MH}$ et al. Prophylaxis with oral penicillin in children with sickle-cell anemia. A randomized trial. New England journal of medicine, 1986, 314:1593-9. 